\title{
A Comparison of Prevention and Treatment of Recurrent Pregnancy Loss with Vaginal Progesterone or Oral Dydrogesterone
}

\section{Schindler $\mathrm{AE}^{\star}$}

Institute for Medical Research and Education, University Clinic, Essen, Germany

${ }^{*}$ Corresponding author: Schindler AE, Institute for Medical Research and Education. University Clinic

Hufelandstrasse 55, SHH1, App.0809, D-45122 Essen, Germany, Fax: +49-201-7499533, Tel: +49-2017991833 E-mail: adolf.schindler@uni-due.de

Citation: Schindler AE (2016) A Comparison of Prevention and Treatment of Recurrent Pregnancy Loss with Vaginal Progesterone or Oral Dydrogesterone. J Gynecol Res 2(1): 104. doi: 10.15744/2454-3284.2.104

Received Date: July 13, 2016 Accepted Date: October 25, 2016 Published Date: October 26, 2016

\begin{abstract}
Most recent publications offer the opportunity not only to look at large studies, but also to be able to compare the effect of the use of micronized progesterone or dydrogesterone for prevention of recurrent (habitual) miscarriage.

Micronized progesterone and dydrogesterone have a similar partial effect pattern, which does not induce unwanted effects, when used in pregnancy. Also their major actions regarding implantation, preparation of the endometrium, blood flow increase, spiral artery development, uterine quiescence, cervical rigidity and protection of the semi-allogenic fetus not to be attacked by the maternal immune system, for instance regulating the Th1- and Th2-cytokines to establish and continue to have a dominance of Th2-cytokine action and protection against these effects which are associated with lower circulating progesterone. Both studies under consideration have included women with a history of three or more previous miscarriages and other non-endocrine or endocrine abnormalities have been ruled out.

Under these provisions prevention of recurrent (habitual) miscarriage compared with a placebo group and a control group without a history of recurrent (habitual) miscarriage in a prospective, randomized fashion resulted in the dydrogesterone group in a recurrent (habitual) miscarriage rate of $6.9 \%$, placebo group $16.8 \%$ and the control group $3.5 \%$. The dose was 2 x $10 \mathrm{mg}$ daily starting between $4-8$ weeks of gestation until the 20st weeks of gestation.

In 2015 an even larger study was done with micronized progesterone with a dose of micronized progesterone 2 x 400 mg a day vaginally. This application was started not later than 6 weeks of gestation and ended at 12 weeks of gestation. The live birth rate was not significant between the groups $(65,8 \%$ for micronized progesterone versus $63,3 \%$ placebo(RR1.04; 95\%CI, 0,99-1,15) .

Together with other studies this indicates that dydrogesterone significantly improves pregnancy outcome in contrast to vaginal progesterone in women with recurrent pregnancy loss.
\end{abstract}

Keywords: Progesterone; Dydrogesterone; Recurrent (habitual) miscarriage

\section{Introduction}

Without doubt, progesterone produced by the corpus luteum of the ovary or during pregnancy by the placenta has a wide range of effects to secure a proper fetal development and an uneventful delivery. Major impact of progesterone is achieved such as increased blood-flow towards the uterus, development of functionally intact spiral arteries and development of the secretory endometrium and conversion into decidua. In addition, progesterone controls uterine contractions and maintains a closed cervix. Since the fetus is semi-allogenic, progesterone plays a decisive role for the fact that the mother is not rejecting the semi-allogenic fetus.

In general, progesterone by the corpus luteum and after 8 weeks of gestation by the placenta rises continuously until term. This establishes an optimal milieu for fetal development [1]. The clinical application of micronized progesterone vaginally and dydrogesterone orally leaves a whole set of questions to be answered for the use of vaginal micronized progesterone and oral dydrogesterone.

The main action of micronized progesterone and dydrogesterone are:

1. Action at the endometrium

2. Blood-flow to the uterus 
3. Development of spiral arteries

4. Quiescence of the uterus

5. Cervical closure

6. Impact on the immune system of the pregnant woman (Th1-, Th2-ratio; progesterone blocking factor (PIBF)

7. When to start with the progesterone prevention?

8. What daily dose should be used?

9. How long should progesterone be given?

\section{Material and Methods}

\section{Micronized vaginal progesterone}

Vaginal micronized progesterone (Utrogest) was supplied by Besins Healthcare. The patients were recruited from hospitals located across UK ( 36 sited) and the Netherlands (9sites). Age range is 18 to 39 years.

Participants were randomly assigned in a 1:1 ratio to receive vaginal suppositories containing $100 \mathrm{mg}$ of micronized progesterone twice daily or matched placebo.

\section{Oral dydrogesterone}

Oral dydrogesterone is given at the beginning $40 \mathrm{mg}$ orally and thereafter 2 x $10 \mathrm{mg}$ daily orally are used. Age range is 18-36 years. The participants were randomly assigned to either $20 \mathrm{mg}$ of dydrogesterone daily and placebo.

\section{Results}

\section{Micronized progesterone}

The most recent and largest study with the highest amount of vaginal micronized progesterone revealed the following [2]:

The rate of life birth after 24 weeks of gestation was $68 \%$ in the progesterone group as compared with $63,3 \%$ in the placebo group (RR1,04; 95\%CI 0,99-1,15).

There were no significant differences between the groups in the rates of clinical pregnancies at 6-8 weeks of gestation, ongoing pregnancies at 12 weeks of gestation, ectopic pregnancy, miscarriage, stillbirth and neonatal outcomes.

The frequency of adverse events did not differ significantly between the progesterone group and the placebo group.

In conclusion the trial showed no significant increase in the rate of life birth with the use of vaginal progesterone in the first trimester pregnancy among women with recurrent (habitual) miscarriage [2].

\section{Dydrogesterone}

In 2014 the largest study so far with dydrogesterone in women with recurrent (habitual) miscarriage was done in a single institution. Only women were selected with at least three recurrent miscarriages age 18 to 35 years, confirmation of pregnancy between 4-6 weeks of gestation after ultrasound proof of fetal viability. 560 cases were enrolled, who fulfilled the criteria; the patients were randomly assigned to orally take $2 \times 10 \mathrm{mg}$ dydrogesterone from the time of enrollment (4 to 8 weeks) to 20 weeks of gestation. Of the 360 patients recruited 180 were randomly assigned to dydrogesterone and 180 to the placebo. A third group consisted of 173 pregnant women who were healthy.

The occurance of another miscarriage after three consecutive miscarriages was significantly higher with $16.8 \%$ (29 of 171) in women with recurrent pregnancy loss compared with healthy controls, 3,5\% (6 of 174), $\mathrm{p}<0.001$ ). The risk of miscarriage after 3 miscarriages was 2.4 times higher in the placebo group versus the treatment group (RR 2,4;95\%CI 1,3- 5,9, $<<0,001)$. The decrease of miscarriage in the dydrogesterone group $(16,8 \%$ in the placebo group) was $6,9 \%$ and this was highly significant $(p<0,004)[3]$.

In addition, the treatment group showed a trend towards reducing the numbers of preterm deliveries, low birth weight babies and small -for date babies. This positive effect has been seen under the intake of dydrogesterone only up to 20 weeks of gestation. Continuation of such prevention up to 37 weeks of gestation is therefore indicated in these pregnancies to achieve an overall improvement regarding the outcome of these pregnancies as delineated elsewhere $[4,5]$.

\section{Discussion}

In women with a history of recurrent (habitual) miscarriage all relevant studies with dydrogesterone have demonstrated a significant improvement of pregnancy outcome [6]. In the group taking dydrogesterone $(\mathrm{n}=175)$ compared with the placebo group $(\mathrm{n}=173)$ there was a miscarriage rate of $23,5 \%$ while for the dydrogesterone group $(n=173)$ the miscarriage rate was only $10.5 \%$ (Odds-ratio 0,29 ; $95 \%$ CI $0,13-0,65$ ). 
From all the data in the literature presented and our own experience so far it is obvious that there is a significant preventive effect in women with recurrent (habitual) miscarriage by dydrogesterone [5]. In contrast, the data available for the effect of micronized progesterone in women with recurrent (habitual) miscarriage including the recent large study with a dose increase of $2 \mathrm{x} 400 \mathrm{mg}$ micronized progesterone intravaginally did not reveal any significant impact [2].

If one takes into account the error of Coomarasamy, et al. that in a 2013 Cochrane analysis a positive effect was found, is not correct [2,7]. Based on the data by Wahabi et al in a previous Cochrane Data Base System Review Study 2011 the paper by El-Zibdeh was added where dydrogesterone was used as progestogen [8,9]. This resulted in a significant therapeutic effect of the whole study, but was not based upon micronized progesterone but rather based upon dydrogesterone [7].

\section{Conclusion}

At present, all data available indicate that dydrogesterone protects significantly pregnancies in women with a history of recurrent (habitual) miscarriage, but similar favourable effects have not been obtained with vaginal micronized progesterone even using $2 \mathrm{x}$ $400 \mathrm{mg}$ daily up to 12 weeks of gestation [2].

\section{Reference}

1. Schindler AE (2004) First trimester endocrinology: Consequences for diagnosis and treatment of pregnancy failure. Gynecol Endocrinol 18: 51-7.

2. Coomarasamy A, Williams E, Truchanowicz E, Seed P, Small R, et al. (2015) A randomized trial of progesterone in women with recurrent miscarriages. N Engl J Med 373: 2141-8.

3. Kumar A, Begum N, Prasad S, Aggarwal S (2014) Oral dydrogesterone treatment during early pregnancy to prevent recurrent pregnancy loss and its role in modulation of cytokine production: a double-blind, randomized, parallel, placebo-controlled trial. Fert Steril 102: 1357-63.

4. Schindler AE (2010) Progestogens for treatment and prevention of pregnancy disorders. Horm Mol Biol Clin Investig 3: 453-60.

5. Schindler AE (2016) Future concepts of prevention or treatment with dyrogesterone in pregnancy disorders. Horm Mol Biol Clin Investig [In press].

6. Carp H (2015) A systematic review of dydrogesterone for the treatment of recurrent miscarriage.Gynecol Endocrinol 31: 422-30.

7. Haas DM, Ramsey PS (2013) Progestogen for preventing miscarriages. Cochrane Data Base syst Rev 10: CD003511.

8. Wahabi HA, Fayed AA, Esmaeil SA, Al Zeidan RA (2011) Progestogen for treatment of threatened miscarriage. Cochrane Data Base syst Rev 12: CD005943.

9. El-Zibdeh MY (2005) Dydrogesterone in the reduction of recurrent spontaneous abortion. J Steroid Biochem Molec Biol 97: 421-5.



